\title{
Un siècle d'infamie. Jalons pour une histoire politique du « Romani cinema »
}

An Infamous Century. Outlines for a Political History of "Romani Cinema"

Jonathan Larcher

\section{OpenEdition}

Journals

Édition électronique

URL : http://journals.openedition.org/recherchestravaux/1113

DOI : 10.4000/recherchestravaux.1113

ISSN : 1969-6434

Éditeur

UGA Éditions/Université Grenoble Alpes

Édition imprimée

ISBN : 978-2-37747-065-5

ISSN : 0151-1874

Référence électronique

Jonathan Larcher, « Un siècle d'infamie. Jalons pour une histoire politique du « Romani cinema » », Recherches \& Travaux [En ligne], 93 | 2018, mis en ligne le 26 octobre 2018, consulté le 08 septembre 2020. URL : http://journals.openedition.org/recherchestravaux/1113; DOI : https://doi.org/10.4000/ recherchestravaux.1113

Ce document a été généré automatiquement le 8 septembre 2020

(c) Recherches \& Travaux 


\title{
Un siècle d'infamie. Jalons pour une histoire politique du « Romani cinema »
}

\author{
An Infamous Century. Outlines for a Political History of "Romani Cinema"
}

Jonathan Larcher

1 Tout au long du $\mathrm{xx}^{\mathrm{e}}$ siècle, la rencontre des familles romanis européennes avec le pouvoir a pris la forme d'une grande variété de persécutions, de relégations, de discriminations et de destructions. Ces diffamations, qui trouvent leur genèse dans la production d' " un régime visuel de l'exclusion ${ }^{1}$ ", à la fin du XIX ${ }^{e}$ siècle, se sont traduites par l'identification, le fichage, l'expertise raciale, l'ingénierie sociale et l'enfermement de centaine de milliers de Roms et Tsiganes, avant qu'ils ne soient déportés et mis à mort lors de la Seconde Guerre mondiale. Cette entreprise de destruction massive, réalisée en grande partie par les collectivités locales et les administrations régionales, se doubla d'une amnésie collective profonde, écartant ainsi le génocide des Tsiganes des procédures de compensation et des premières historiographies sur le nazisme et les camps de concentration ${ }^{2}$. Par cette double opération d'effacement qui le caractérise, le Samudaripen (le «meurtre de masse » en romanès) constitue l'expérience fondamentale à partir de laquelle il est possible de juger d'un « cinéma des infâmes » qui s'attache aux expériences des Roms et Tsiganes.

Dans le cadre d'une recherche en cours sur le « Romani cinema ", conçu à l'intersection entre les cinématographies d'avant-garde et les familles romanis (Roms, Manush, Gitanos, Gypsies, Travellers, Sinti, Zingari) ${ }^{3}$, cet article s'attache à quatre films qui ont marqué d'une pierre blanche l'histoire politique et visuelle des présences tsiganes au cinéma. C'est dans les franges et les marges des cinématographies de l'Europe centrale ou des Balkans, plutôt que parmi les filmographies thématiques sur les Roms et Tsiganes, ou les "gypsy films ${ }^{4}$ ", qu'apparaissent ces fragments qui marquent un siècle d'infamie. À quelques exceptions près, aucun des quatre films discuté ici n'est répertorié dans les filmographies consacrées aux Roms et Tsiganes qui fleurissent en ligne ou composent les corpus des publications sur les représentations visuelles des Roms. C'est 
donc parmi les fragments filmiques, parfois touchés par la censure, très souvent rétifs aux industries culturelles nationales dans lesquels ils s'inscrivent, ou bien même sauvés des archives, que ce siècle d'infamie au cinéma est observable, sous l'angle à la fois d'une archéologie et d'une agentivité des images filmiques. J'entends ici par "agentivité des images", leur fonction au moment précis de leur réalisation, ce qu'elles font et ce qu'elles font faire. À l'instar de ce que Michel Foucault avait justement observé dans son travail parmi les archives, ces fragments de présences tsiganes dans les arts filmiques sont irréductibles à leur authenticité ou « leur valeur représentative » :

J'ai tenu à ce que ces textes soient toujours dans un rapport ou plutôt dans le plus grand nombre de rapports possibles à la réalité : non seulement qu'ils s'y réfèrent, mais qu'ils y opèrent qu'ils soient une pièce dans la dramaturgie du réel, qu'ils constituent l'instrument d'une vengeance, l'arme d'une haine, un épisode dans une bataille, la gesticulation d'un désespoir ou d'une jalousie, une supplication ou un ordre $^{5}$.

3 Les quatre films présentés et analysés au sein de cet article montrent toutefois les déplacements opérés par la présentation de ces vies infâmes par les moyens des arts filmiques. Frappé par « ces vies infimes devenues cendres dans les quelques phrases qui les ont abattues ${ }^{6}$ ", Michel Foucault compose sa recherche et son écriture à partir de traces et de textes. Loin de constituer une matière exogène à l'expérience vécue de ces individus, ces « fragments de discours » recueillis dans les archives constituent autant de «fragments d'une réalité dont ils font partie ${ }^{7}$ ». Comme le précise très bien le philosophe et historien, "des vies réelles ont été "jouées" dans ces quelques phrases [...] de fait, leur liberté, leur malheur, leur mort souvent, leur destin en tout cas y ont été, pour une part au moins, décidés ${ }^{8} "$.

4 L'inscription de ces fragments d'archives dans l'expérience vécue des vies infâmes distingue le travail de Michel Foucault de celui des cinéastes dont il sera question dans les lignes qui suivent. À l'exception du remploi d'archives filmiques opéré par Charmant Rouge, le groupe de musiciens autrichiens auteur de Fingerprints (2001-2007), les images réalisées par ces cinéastes d'avant-garde arrivent après, bien après, la rencontre des familles romani avec le pouvoir. Si ces images enregistrées présentent l'expérience historique de ces familles, elles empruntent la plupart du temps le détour de la farce, du témoignage, du détournement ou de la reconstitution mobilisant un répertoire figuratif qui sera déployé au fil du texte. En dépit d'une présentation des films dans l'ordre chronologique de leur réalisation, cette archéologie du « Romani cinema » fait d'incessants va-et-vient entre les catastrophes qui ont marqué le siècle d'infamie des Roms et Tsiganes au cinéma'.

\section{La farce politique. - L'histoire qui se répète (Zbehovia a pútnici, Jakubisko, 1968)}

5 En apparence, il n'y a probablement pas de films aussi éloignés des "gypsy films » et des films sur le génocide ou les catastrophes vécues par les Roms que Zbehovia a pútnici (Deserter and the Nomads ou Deserter and the Pilgrims), réalisé et conclu en 1968 alors que l'armée du pacte de Varsovie envahit les rues de Prague. Cuuvre de fiction, à mi-chemin entre le film de patrimoine, le poème visuel, et le cinéma d'anticipation, le film de Juraj Jakubisko ne reprend ni le chatoiement des chromatismes propres aux compagnies bohémiennes (ou gitanes), ni ne présente des expériences historiques ou quotidiennes 
idiomatiques des Roms et Tsiganes. Dans son second long-métrage, le réalisateur slovaque dépeint en trois segments d'une trentaine de minutes chacun les conséquences des deux guerres mondiales et d'une catastrophe nucléaire sur le monde rural de la Tchécoslovaquie. Personnage central, « La mort » traverse les trois « récits » en donnant à chacun d'eux l'aspect d'une farce grotesque sur les ressorts de la guerre et de la destruction. Dans un monde rural marqué par le folklore et le fantastique, Juraj Jakubisko propose deux figurations inédites ${ }^{10}$. À rebours des imageries fantastiques ou néogothiques des Bohémiens et "Tziganes de l'Est», la communauté romani de Zbehovia a pútnici n'est nullement l'incarnation d'une explication irrationnelle des faits ou la face avenante d'une réalité monstrueuse encore cachée ${ }^{11}$. Par conséquent, l'intrigue du premier segment du film inscrit les familles tsiganes et les paysans gadjé (non-roms) dans une même communauté de destin.

6 Zbehovia a pútnici s'inscrit dans la «nouvelle vague » du cinéma tchécoslovaque qui s'étend de 1962 jusqu'au printemps 1969, quand les effets de l'invasion soviétique un an plus tôt commencent à affecter l'industrie cinématographique. Tourné et monté en 1968, au cœur de ces deux séquences historiques, le film de Juraj Jakubisko garde la trace de ce mouvement cinématographique, qui est un véritable coup de force esthétique, tout en accueillant dans son récit des images qui documentent l'arrivée des avions de combats et des tanks de l'armée soviétique dans les aéroports et les rues de Prague. Ces séquences qui interrompent le second récit du film qui vient juste de présenter la fin de la Seconde Guerre mondiale sont accompagnées de quelques mots lus en voix off:

Nous avons considéré le premier tank que nous avons vu comme une obligation à réaliser un autre film. Notre temps est aussi fou que le passé, et peut-être bien que le futur. Notre espoir de mettre un terme à tout cela est seulement un rêve que l'on peut imaginer. Motivés par notre impuissance, nous avons décidé de finir le film. Bien que nous ne sachions pas si quelqu'un verrait un jour le film et si les temps qui viennent nous permettront de raconter une histoire librement. [Quelques photographies du tournage s'intercalent entre les images de l'invasion]. Il y a parmi eux des acteurs qui interprètent des morts quand, dans les rues, il y a de véritables victimes qui n'ont nullement besoin de simuler quoi que ce soit. Continuons l'histoire. Imaginons que la [Seconde] Guerre [mondiale] est vraiment finie.

7 Par un effet d'anticipation, l'intégration de ces séquences sur l'invasion soviétique provoque de fait la censure du film et un coup du sort fait disparaître les négatifs. Ce n'est qu'au début des années 2000 , qu'une copie retrouvée permet de projeter à nouveau le film dans sa version d'origine.

8 Le premier des trois films, Zbehovia (Deserter ou Deserters), narre les défections de Kalmán, un soldat tsigane et de Martin, un révolutionnaire bolchévique ${ }^{12}$. Engagés dans l'armée austro-hongroise, ils désertent, par conviction politique ou par peur de rencontrer (à nouveau) la mort ${ }^{13}$. La structure du récit repose ainsi sur les fuites et pérégrinations des deux protagonistes pour échapper aux recherches des soldats. Cet arc narratif et ces poursuites le plus souvent grotesques sont sans cesse interrompus par des explosions visuelles figurées par l'usage de pellicule inversible, par des séquences oniriques tournées caméra à l'épaule ou par l'insertion au montage de photographies documentaires.

9 Au gré des passages entre films négatifs et positifs, des accélérations du défilement de la pellicule et des prises de vue aux angles déformants, la campagne se transforme en un univers macabre. Ainsi la romance entre Kalmán et Lila, la jeune tsigane, qui 
emprunte l'ensemble de ses motifs au régime visuel de la pastorale, est constamment transformée en cauchemar par ces renversements chromatiques qui présentent les visions du déserteur hanté par la venue imminente de la mort.

La confrontation du monde tsigane à cet univers fantastique engendre des représentations inédites. Si la croyance en des forces surnaturelles est toujours propre aux Tsiganes, elle est néanmoins dotée de sens, comme lorsque Kalmán se brûle les mains pour se laver du sang versé pendant la guerre. Inversement, l'attitude du capitaine des hussards qui salue et accueille la mort au banquet de son propre mariage présente toute l'absurdité d'une société en état de guerre.

11 Toutefois, malgré la clairvoyance de Kalmán et la parfaite intégration des musiciens tsiganes dans le monde rural, l'ultime rebondissement du récit transforme la célébration du mariage en une révolution burlesque, aussitôt avortée pour aboutir à une farce sanglante. Les noces morbides et grotesques célébrées à la fin du film, autour des cadavres de Kalmán et de son ami Martin, figurent, de manière inédite dans l'histoire du cinéma fantastique, le Tsigane comme une victime.

Bien davantage encore, et c'est là le premier jalon posé par Zbehovia a pútnici dans l'histoire politique du "Romani cinema ", les images de cette noce macabre entre le tsigane et le révolutionnaire, célébrée par l'ensemble de la société villageoise, annoncent et préfigurent le destin tragique que ces populations (de Tsiganes et dissidents politiques) connaîtront avant et après la Seconde Guerre mondiale. Un horizon suggéré par le texte qui introduit le second segment du film : « quand les gens s'entretuent par haine, c'est terrible... mais c'est encore pire quand ils apprennent à le faire mécaniquement $»$.

13 Par la représentation de ces personnages tsiganes unis en une même communauté de destin que les Gadjé qui les entourent, Juraj Jakubisko réalise une composition singulière, contrastant avec l'ensemble des représentations cinématographiques de l'histoire du cinéma européen, qui incluent très souvent les Roms et Tsiganes dans le champ de l'image pour se focaliser sur les relations contrastées ou conflictuelles entre " eux » et les Gadjé. Dans Ružové Sny (Rêves en rose, 1976), un très beau film réalisé au sein d'une communauté rom quelques années plus tard, le cinéaste slovaque Dušan Hanák reprend une nouvelle fois cet éternel problème des relations entre Tsiganes et Gadjé.

\section{La voix. - Témoignages de la catastrophe (Zigeuner sein, Nestler, 1970)}

14 Alors que la stupeur déclenchée par la Shoah engendra des procédures juridiques et la mise en place de compensations pour les victimes juives, le sort des familles romanis internées et envoyées dans les camps de la mort fut différent. "Pendant des années après la fin de la Seconde Guerre mondiale, beaucoup de Tsiganes victimes des nazis militèrent, certains jusqu'à leur mort, pour la reconnaissance des torts subis, ainsi que pour être dédommagés financièrement pour tout ce qu'ils avaient perdu. Pour la plupart, l'entreprise fut vaine ${ }^{14}$.»

L'asymétrie entre l'Holocauste et le Samudaripen est également visuelle. L'image de la petite fille filmée depuis le quai du camp de transit nazi de Westerbork, la tête penchée dans l'entrebâillement de la porte du wagon qui l'emmène vers les camps de la mort, 
est déjà une icône de la Shoah. Une image que nous connaissons en partie grâce à Nuit et brouillard d'Alain Resnais (1956). Il faudra toutefois attendre le milieu des années 1990 pour que la petite fille retrouve son nom et son identité. Settela était une petite Sinti ${ }^{15}$. Dans le silence et le désert visuel qui entourent le génocide des Tsiganes au lendemain de la Seconde Guerre mondiale, deux cinéastes allemands produisent un film documentaire inédit sur l'expérience des survivants roms et sintis de l'Allemagne de l'Ouest et de l'Autriche. En tout juste quarante-sept minutes, Peter et Zsóka Nestler présentent une série de témoignages sur la montée de l'anti-tsiganisme avant la guerre, la vie dans les camps et la perpétuation d'une marginalisation de leur famille dans les franges urbaines.

Zigeuner sein de Peter et Zsóka Nestler s'inscrit dans le contexte du Nouveau cinéma ouest-allemand qui émerge au cours des années 1960 et 1970. Par la présentation, frontale et sans ornements, des récits de vie des Sinti, Zigeuner sein appartient à ce mouvement qui produit des «représentation[s] critique[s] d'un pays qu'on ne peut plus, après le nazisme, nommer Heimat [patrie] ${ }^{16}$ ». Ce regard critique n'est rendu matériellement possible que par l'exil de Peter et Zsóka Nestler en Suède. Zigeuner sein est en effet produit par la Radio Suédoise (Sveriges Radio).

17 Tout au long du film, le montage alterne entre des prises de vues des lieux parcourus par les cinéastes, et habités par les familles tsiganes, et des entretiens avec les survivants des camps. Deux longues séquences dérogent quelque peu à cette règle. La première, qui ouvre le film est une série de toiles du peintre Otto Pankok, réalisées lors de son séjour de quatre ans dans une communauté tsigane et présentées devant l'objectif de la caméra par Zsóka Nestler. Le défilement des toiles, parcourues sans quasiment aucune coupe, est accompagné par une voix-off, qui présente brièvement la longue histoire des persécutions endurées par les familles tsiganes depuis plusieurs siècles et décennies en Allemagne. La seconde séquence se situe au milieu du film et présente les registres du camp tsigane d'Auschwitz-Birkenau, retrouvés plusieurs années après guerre. Avec une économie de commentaires, ces images présentent les noms des détenus, leur matricule, et quelques clichés photographiques pris à leur arrivée dans le camp.

Si ces séquences sont en tout point inédites, Zigeuner sein est particulièrement remarquable pour les témoignages qui constituent la plus grande partie du film. En effet, l'écoute des cinéastes et leur attention portée à la voix des personnes filmées sont en tout point exemplaires. Lors des deux premiers entretiens avec des survivants des camps, le regard des sujets filmés est dirigé hors champ, très probablement en direction de Zsóka Nestler, à la prise de son. L'ensemble des prises de vues se fait depuis la même position, Peter Nestler filmant ses interlocuteurs de trois-quarts ou de profil. Les silences gardés au montage ou les sautees entre les plans induites par chaque coupe donnent ainsi l'impression que les récits sur les problèmes de santé, les difficultés d'indemnisation, les insomnies et les peurs sont énoncés dans un seul souffle. Sans fioritures, ce léger retrait du cinéaste, qui évite toute forme de frontalité ou de mise à distance confortable, nous positionne, en tant que spectateur, sur le seuil du dialogue silencieux engagé entre les personnes filmées et Zsóka Nestler, toujours hors champ. Cette prudence fait rapidement sens lorsque la femme d'un survivant refuse d'être filmée, se tenant au fond du champ, dans l'ombre de la cuisine. Rencontrer les familles tsiganes chez elles, quelques décennies après la guerre, s'avère en effet une situation délicate pour une équipe de tournage. Contrairement aux décisions émanant de 
l'appareil d'état nazi qui ont conduit au génocide des Juifs, le génocide des Tsiganes fut une entreprise élaborée à l'échelle du local: «[...] dans les bureaux des racistes d'université ou de conseil municipal, dans les cellules de la police criminelle de Francfort ou de Munich [et] sur les parcelles des camps municipaux obligatoires de la Ruhr où les familles tsiganes recevaient la visite de scientifiques racialistes ${ }^{17}$ \%. Avec beaucoup de tact, Peter Nestler et Zsóka Nestler mettent donc en place des situations de tournage qui s'adaptent à «une culture de la persécution [qui] se transmet silencieusement de génération en génération ${ }^{18}$ ».

19 Ces précautions ne relèvent donc ni de maniérismes ni de « peurs de filmer ». En effet, dès lors qu'il s'agit de filmer les scènes extérieures, la caméra de Peter Nestler se laisse facilement guider par les adresses des enfants et des parents qui sourient en direction de la caméra. De la même façon, c'est avec une toute autre frontalité que les cinéastes filment l'auteur Hermann Langbein, ancien détenu de droit commun et qui fut un témoin privilégié des conditions de vie déplorables des Tsiganes et des Roms à Birkenau. Lors de cet entretien, la caméra est sur pied, face à au sujet filmé, à deux ou trois mètres de lui. Le micro posé sur la table est dans le champ de l'image.

Zigeuner sein pose deux jalons pour une étude des présences tsiganes et roms dans ce long siècle d'infamie. Le film de Peter et Zsóka Nestler marque tout d'abord d'une pierre blanche l'histoire visuelle du génocide tsigane. Par l'attention portée aux témoignages - leur immédiateté, leur rythme, leur souffle - les cinéastes montrent toute la capacité des victimes tsiganes à présenter leur vie et mettre en mot leur expérience ${ }^{19}$. En réalité, l'exil de Peter et Zsóka Nestler n'est pas seulement une caractéristique du Nouveau cinéma allemand des années 1960 et 1970. Il est aussi l'un des aspects persistants du «Romani cinema» depuis la Seconde Guerre mondiale, comme s'il était nécessaire pour ce cinéma de se former en dehors de la société de voisinage que côtoient les Roms et Tsiganes. Qu'il soit accueilli avec moins de méfiance ou qu'il soit des imageries et archétypes qui peuplent l'imaginaire des gadjé, c'est bien souvent le regard de l'étranger qui se porte en premier vers les familles roms et tsiganes. Ainsi, le premier film documentaire et ethnographique sur les Manush français est réalisé par le couple d'anthropologues japonais Yasuhiro Omori et Kimie Omori Mour Djiben. Ma vie de tzigane manouche (1976). Deux décennies plus tard, les films les plus marquants sur les bidonvilles de Roms roumains en France sont aussi caractérisés par ce regard éloigné. C'est aussi bien le cas de Qui a peur des Tziganes roumains? (1996) coréalisé par le photographe et anthropologue argentin Leonardo Antoniadis et la cinéaste Evelyne Ragot, que de France 2007 (2007), réalisé avec des bobines de Super 8 périmées par le vidéaste et plasticien coréen Gee-Jung Jun.

\section{L'assemblage. - Détournements, censure et apparition des Roms dans l'espace public grec (ROM, Karamaghiolis, 1989)}

21 À la fin des années 1980, Menelaos Karamaghiolis réalise un long métrage de commande pour la télévision grecque, qui lui concède un documentaire sur les Roms en Grèce, après le relatif succès rencontré par son film précédent sur l'île de Lesbos. Dans une économie qui confine le cinéma documentaire à la télévision et n'accorde que peu de moyens financiers pour de tels films, le cinéaste démarre le tournage en filmant les Roms qu'il côtoie et rencontre dans la frange urbaine qui environne Thèbes, où il réside. Dans l'impossibilité de tourner l'ensemble du film avec une équipe, Menelaos 
Karamaghiolis met ainsi en place un cinéma de bout de ficelles, combinant des photographies réalisées seules, des images en mouvement, la plupart du temps non synchrones, et des enregistrements sonores de conversations.

Avec une économie de moyens extraordinaire, le cinéaste relève un double défi. Il s'agit tout d'abord de présenter l'expérience et l'histoire des Roms au public grec, et ainsi de clarifier quelques malentendus à une époque où le terme n'est employé ni par les intéressés ni moins encore par les Gadjé. Complémentairement, le défi est aussi formel. Dans un espace médiatique qui limite le film documentaire aux reportages télévisuels, Menealos Karamaghiolis relève le pari de composer une forme qui puisse être projetée en salle. Le pari est effectivement gagné puisque ROM est non seulement le premier film documentaire à être distribué nationalement en salle mais rencontre également un franc succès au Festival de Cinéma de Thessalonique de 1989, participant ainsi à l'émergence du terme « rom » dans l'espace public grec.

$\mathrm{Du}$ tournage au montage, Menelaos Karamaghiolis compose ainsi une véritable mosaïque, par l'ajout de voix offs et de morceaux de musique, dont une composition originale qui constitue un véritable leitmotiv. Cette pratique de l'assemblage d'éléments aussi hétéroclites constitue ainsi le troisième jalon de cette histoire du «Romani cinema ». ROM prend ainsi de court les conventions de la fresque qui, dans son aspect historique, comme panoramique, hante l'ensemble des "gypsy films": depuis les grandes célébrations baroques de Tabor ukhodit $v$ Nebo (Les Tsiganes montent au ciel, Loteanu, 1976) ou Dom za vešange (Le Temps des Gitans, Kusturica, 1988), jusqu'aux formes épiques de Latcho Drom de Tony Gatlif (1993). Les continuités propres à l'une et l'autre convention de la fresque opèrent bien souvent des réductions et raccourcis. La première transforme la communauté des vivants en paysage, quand la seconde produit une linéarisation de l'expérience historique des groupes romanis. C'est exactement ce que fait Tony Gatlif en présentant une série de vignettes sur la musique et la danse des Roms et Tsiganes qui habitent aujourd'hui sur la route empruntée par les familles romanis des siècles plus tôt, du Rajasthan à l'Espagne, en passant par la Turquie, la Roumanie, la France, etc.

L'assemblage formé par ROM provoque quelques vives réactions parmi les commanditaires et producteurs du film. L'ERT [Ellinikí Radiofonía Tileórasi - la Radio Télévision Hellénique] va essentiellement découper le son, sans pour autant rééditer les images, montrant ainsi l'important contrepouvoir que représente la voix des Roms et Tsiganes, tout en conservant d'autres éléments tout aussi critiques, non relevés par les censeurs. La genèse de Rom, résumée ici en quelques lignes par Menelaos Karamaghiolis, révèle toute la pertinence des formes élaborées par le cinéaste. Elle montre également comment le film décrit à la fois la situation des familles roms et les idéologies prévalant dans les arènes médiatiques et politiques grecques à la fin des années 1980.

L'ERT a exercé une sorte de censure sur le film à une époque où les documentaires étaient sous-estimés en Grèce. Une personne de l'ERT est venue et a dit: «Nous sommes les producteurs et nous voulons voir le film avant qu'il ne soit terminé ». Après l'avoir vu, elle a dit : «Le film ne peut pas être laissé tel quel, nous devons couper tous les entretiens où les Roms demandent des écoles, de l'aide, la justice, des biens ». Nous avons aussi dû couper un conte de fées rom et une chanson qui était très populaire à l'époque, un Rom qui chantait que « la Madone me regarde en larmes... », l'ERT ne l'a pas acceptée non plus. Mais ils n'ont pas compris que je trichais beaucoup sur ce qui est grec et ce qui ne l'est pas, parce que vous savez, nous avions (et nous avons toujours) ces discours très dangereux sur ce qui est grec, 
ce que nous héritons de la Grèce antique. Bien sûr, nous parlons la même langue, mais nous devrions d'abord apprendre ce qu'était la civilisation grecque antique, et ensuite dire si nous en sommes ou pas la continuation. Même durant l'Empire Byzantin, la Grèce n'existait pas comme pays. C'est un pays qui a deux siècles. Voilà la raison pour laquelle j'ai fait ce jeu avec deux garçons roms portant des costumes nationaux grecs pour une séance photo. Pour moi, c'est cela, le scandale du film... et personne ne l'a remarqué. Si le film était réalisé aujourd'hui, avec Aube Dorée, ils l'interdiraient. Vous avez deux Roms portant ce que nous croyons représenter la quintessence de l'identité nationale grecque ${ }^{20}$.

Si bien sûr le contexte politique de l'époque a permis la diffusion de telles images subversives, leur passage entre les mailles de la censure s'explique probablement aussi par la très grande densité de l'assemblage opéré par Menelaos Karamaghiolis, faisant surgir à chaque visionnage de nouveaux effets de sens.

Composant avec la contrainte d'une équipe limitée à quelques jours de tournage, Menelaos Karamaghiolis réalise toutes les images possibles et s'adapte au désir de cinéma de ses interlocuteurs. Complémentairement aux séquences filmées présentant la vie quotidienne dans les campements en marge des villes ou le travail saisonnier dans l'agriculture, le cinéaste réalise également des images qui correspondent à celles que les Roms veulent offrir d'eux-mêmes. Beaucoup de séquences du film sont ainsi réalisées en accord avec les pratiques vernaculaires de l'image, qui prévalent sur place, comme les photographies de mariage et d'enterrements, ou les « films de commande familiaux » qui «fonctionnent dans le cercle domestique » mais sont toujours réalisés par des professionnels ${ }^{21}$. De la même façon, les images du pèlerinage à Tinos, qui constituent l'une des séquences les plus fortes du film, sont réalisées pour partie sans aucune équipe, le cinéaste suivant simplement les suggestions et les invitations des familles roms pour qui la fête de l'Assomption est le moment le plus marquant de l'année. Ces images, mêlant des formats différents, des situations aussi diverses et les désirs de cinéma du cinéaste et des sujets filmés, ne tiennent ensemble que par un prodigieux travail de montage. Par l'importance accordée à l'éclat, aux silhouettes, aux ombres, aux reflets et aux regards caméra, les points de vue qui semblent prévaloir à la réalisation de ces images sont ainsi mis en regard.

Cette correspondance est également mise en œuvre par l'entremêlement des voix off qui composent l'écologie sonore du film. Dans la veine d'un Chris Marker, Menelaos Karamaghiolis orchestre quatre voix off qui, sur des registres de paroles différents, tiennent des propos parfois opposés :

Tout d'abord nous avons besoin de connaître l'histoire des Roms : c'est la voix du professeur. Ensuite, nous devons aussi connaître leur conte, nous avons donc besoin d'une veille femme rom [Tamara]. Ensuite, nous avons besoin de notre point de vue, ce qui correspond au photographe qui réalise les clichés. Et nous avons besoin de leur propre voix, celle de la jeune Rom, qui, et c'était mon idée, est une fille qui cache le fait qu'elle soit tsigane [Aima] ${ }^{22}$.

28 L'ensemble des quatre voix se combine avec des lectures de l'œuvre de Kostís Palamás, poète et homme de lettre grec, auteur notamment du recueil $O$ dōdekálogos tou gýftou (Les douze paroles du Tzigane, 1907). En évitant les clichés et poncifs qui peuvent être attachés à des œuvres de l'avant-garde qui confondent le documentaire et le conte, le cinéaste parvient à détourner le sens initial des images en leur donnant une profondeur historique (ou légendaire) ${ }^{23}$. Par le récit des diffamations subies par les populations roms, le sens des images d'enterrement et de pèlerinage à Tinos, avec son cortège de policiers, d'hommes religieux et de politiciens, est détourné. Cette mosaïque d'images 
figure alors ces évènements narrés par la voix off et dont il n'existe aujourd'hui que pas ou peu d'images enregistrées (en dehors des fonds archives, pour les plus récents).

Par son souci de composer avec le désir de cinéma de ses interlocuteurs, en donnant à ses images l'aspect des images vernaculaires réalisées sur commande, Menelaos Karamaghiolis crée ainsi une véritable mosaïque de regards et de points de vue, sans pour autant se dédouaner de ses responsabilités d'auteur. Bien qu'il ne soit pas allé jusqu'à confier la caméra ou l'appareil photographique à ses interlocuteurs, Menelaos Karamaghiolis est probablement l'un des premiers cinéastes de l'histoire du « Romani cinema » à avoir conçu son film avec une conscience aussi aiguë du savoir des images que possèdent ses interlocuteurs et de l'écologie des images, des récits et des discours qui entourent les familles roms.

\section{La chose même. - Reprise des images et des technologies de l'infamie (Fingerprints, Charmant-Rouge, 2001-2007)}

Avec toutes les réserves que nécessite une recherche en cours, il semble donc qu'il faille attendre le début du $\mathrm{xx}^{\mathrm{e}}$ siècle pour que des cinéastes - en l'occurrence les musiciens et vidéastes du groupe Charmant Rouge - prennent en main les archives filmiques qui ont été réalisées dans le moment de confrontation le plus intense entre les familles romanis et le pouvoir. En une série de séquences d'archives filmiques, Fingerprints (2001-2007) présente l'asymétrie et la violence inhérentes aux rencontres entre les Tsiganes et l'élite économique puis la bureaucratie du Burgenland (la province la plus à l'Est de l'Autriche), au cœur des années 1920 et 1930.

31 Fingerprints est un ready-made composé exclusivement des séquences tirées des Archives Autrichiennes du Film. Remontées avec une composition sonore écrite par deux membres du groupe (Andreas Berger et Robert Pinzolits ${ }^{24}$ ), les images d'archives montrent comment les technologies filmiques furent dévolues au renforcement de la souveraineté de l'État sur les nouveaux territoires du Burgenland et sur les corps des Roms et Sinti de la région, à la veille de la Seconde Guerre mondiale.

32 Libre de droits, les matériaux d'origine (pellicule) ont ainsi été transférés en VHS en 2001, à l'occasion du $80^{\mathrm{e}}$ anniversaire de l'entrée du Burgenland dans l'État autrichien. Le groupe Charmant Rouge se saisit de ces images la même année, à l'occasion d'un workshop d'une semaine, qui réunit dans un centre culturel du Burgenland des dizaines d'artistes (photographes, musiciens, peintres, sculpteurs) dans l'objectif de produire des œuvres qui seront présentées en conclusion de l'événement. En raison de l'anniversaire de la région, le workshop devient également une scène politique. Des commissaires du gouvernenement européen et des responsables politiques locaux assistent à la présentation des travaux qui concluent le workshop. Comme le précise David Kleinl, membre du groupe Charmant Rouge et monteur du film Fingerprints, cette édition s'inscrit également dans un contexte politique qui rend d'autant plus urgente la présentation des archives filmiques montrant un jeune zigeuner se faire photographier et prendre les empreintes digitales devant l'objectif de l'opérateur.

À cette époque, c'était après le 11 septembre, et des politiciens autrichiens, membres du gouvernement de l'époque, avaient soulevé la possibilité de relever les empreintes digitales de tous les citoyens. Un tel discours était inédit, tout comme cela était très nouveau pour nous de voir ces anciens matériaux où les Roms et Sinti se faisaient prendre les empreintes digitales. Montrer ces images était un premier commentaire adressé aux politiciens de cette époque ${ }^{25}$. 
donc dans ce contexte très particulier que David Kleinl numérise la cassette VHS.

Après avoir sélectionné les segments et ralenti quelque peu la vitesse de défilement des images, le court métrage est transposé sur une mini-DV et projeté lors d'une performance musicale, qui sera plus tard reprise pour constituer de façon pérenne la bande son $\mathrm{du}$ film. La genèse de Fingerprints montre encore une fois l'importance d'élargir le spectre des arts filmiques pour rendre compte du siècle d'infamie de la présence romani au cinéma.

Sans aucun commentaire, le montage de Fingerprints s'organise en trois temps autour de séquences délimitées par des passages au noir. La première séquence, qui ouvre le film, réunit des plans présentant des scènes pastorales et bucoliques. Ces vignettes de quelques secondes montrent les gestes quotidiens des agriculteurs ou les activités plus rituelles de la récolte, de l'épandage du fumier et de l'ensemencement des champs. Quelques images de la construction d'une voie de chemin de fer concluent cette première partie $\mathrm{du}$ film. La seconde séquence, plus longue, présente plusieurs plans filmés à deux occasions. Dans la première série d'images, des familles roms sont filmées déjeunant sur l'herbe autour de leurs roulottes. À cette première scène de repas succède une seconde avec des enfants roms qui jouent et se disputent sur le bas-côté de la route. Dans le coin gauche du cadre apparaît le phare avant d'un bus, depuis lequel sont tournées les images. En effet, ces images ont été réalisées par des participants à des visites touristiques organisées au cours des années 1920 et 1930 en Autriche, dans l'objectif que les citoyens puissent mieux connaître leur pays, et en particulier cette région du Burgenland, la plus pauvre de l'état autrichien.

Suite à ces imageries touristiques apparaissent les images tournées au sein de l'administration locale montrant un jeune sinti se faisant photographier et relever les empreintes digitales. Cette séquence constitue plus de la moitié du film. Bien que les images ne soient pas datées ou contextualisées, elles font sûrement suite à la mesure prise en 1925 par l'administration du Burgenland pour constituer un fichier de tous les Tsiganes vivant dans la région ${ }^{26}$. Avec une grande précision, l'opérateur filme l'ensemble de la procédure et des gestes qui permettent la constitution d'une fiche de renseignement: photographies, relevés de tous les doigts séparément, puis de l'ensemble des phalanges supérieures des doigts de la main gauche et droite sans les pouces, et, enfin, classement des fiches (probablement par ordre alphabétique). "Romani cinema ». Fingerprints montre tout d'abord des formes de continuités entre une pratique bourgeoise et amateur des technologies filmiques et une utilisation bureaucratique de ces mêmes technologies. C'était en effet majoritairement des touristes des classes supérieures qui effectuaient ce grand tour de l'Autriche. En ce sens, la succession de ces vignettes bucoliques et de mise en fiche des individus roms mettent en image ce que Michael Stewart a justement qualifié, à propos du Burgenland, d'« anti-tsiganisme pastoral ${ }^{27} »$.

Enfin, ces images de Fingerprints montrent dans le détail le corps à corps qui se joue entre l'autorité et des vies infâmes sur la scène du pouvoir, tout en constituant la seule trace indélébile de leur existence. La très grande partie des Tsiganes du Burgenland mourra en déportation. Dans «le canton d'Oberwart [...], en 1946, le poste de gendarmerie locale estimait que sur les quelques 3000 Tsiganes que comptait l'ensemble de la population cantonale, à peine 200 étaient rentrés chez eux ${ }^{28}$ ». 


\section{Conclusion}

Tout au long de cet article, j'ai fait le choix de ne pas m'étendre sur l'analyse des conditions historiques, sociales et politiques de réalisation des films, pour davantage rendre compte des enjeux formels qui constituent les jalons de ce siècle d'infamie des Roms et Tsiganes au cinéma.

La présentation du contexte des œuvres, nécessaire compte tenu du fait que la plupart d'entre elles ont été peu distribuées en France, a montré combien il faut chercher ces portions de vies et d'expériences dans les franges des arts filmiques (censure, exil, oubli ou ready-made).

En ce sens, les quatre films ne forment pas seulement les jalons d'une fresque chronologique à recomposer. Ils le sont en partie bien sûr. Les expériences infâmes vécues par les Roms et Tsiganes d'Europe que les trois premiers films ont figurées par la farce, la parole, ou la fabulation, les archives filmiques exposées par Charmant Rouge les ont révélées dans leur forme de "poèmes vies", avec "le désordre, le bruit et la peine, le travail du pouvoir sur les vies, et le[s images qui en naissent $]^{29} »$. Il est pour autant hors de question de fixer les ressorts figuratifs de ces quatre films sur un schéma linéaire. La farce, la voix, l'assemblage d'images vernaculaires, la mise en regard des registres de discours et la reprise des images elles-mêmes forment une gamme de gestes et de ressources plastiques qui sont des repères essentiels pour une archéologie politique du « Romani cinema ».

\section{BIBLIOGRAPHIE}

\section{Filmographie}

LEONARDO ANTONIADIS ET EVELYNE RAGOT

Qui a peur des tziganes roumains ? (France), 1996, vidéo, coul., son, 68'.

CHARMANT ROUGE

Fingerprints (Autriche), 2001-2007, numérique, n\&b, son, 7’.

MENELAOS KARAMAGHIOLIS

ROM (Grèce), 1989, 16mm, coul, son, 75'.

JURAJ JAKUBISKO

Zbehovia a pútnici / Deserters and Pilgrims, (Tchécoslovaquie/Italie), 1968, 35mm, coul, son, 101'.

GEE-JUNG JUN

France 2007 (France), 2007, 16mm, n\&b, sil., 18’.

PETER NESTLER ET ZSÓKA NESTLER

Zigeuner Sein / Att vara zigenare (Suède), 1970, 16mm, n\&b, son, 47'.

YASUHIRO OMORI ET KIMIE OMORI

Mour Djiben. Ma vie de tzigane manouche (France), 1976, 16mm, coul., son, 59'. 


\section{Bibliographie}

AASMAN Susan, 1995, « Le film de famille comme document historique », oDIN Roger (dir.), Le Film de famille : usage privé, usage public, Paris, Méridiens-Klincksieck, p. 97-111.

ABOUT Ilsen, 2004, « Les fondements d'un système national d'identification policière en France (1893-1914), Anthropométrie, signalements et fichiers ", Genèses, nº 54, p. 28-52.

ABOUT Ilsen, 2014, « Une fabrique visuelle de l'exclusion. Photographies des Tsiganes et figures du paria entre 1880 et 1914 », CoQUio Catherine \& Jean Luc PoueYTo (dir.), Roms, Tsiganes, Nomades. Un malentendu européen, Paris, Karthala, p. 431-446.

ASSEO Henriette, 2014, « Une historiographie sous influence », COQUIO Catherine \& Jean Luc POUEYTo (dir.), Roms, Tsiganes, Nomades. Un malentendu européen, Paris, Karthala, p. 63-82.

ASSEO Henriette, 2016, « Politique de la race et expansionnisme nazi », Études tsiganes, n 56-57, p. 10-27.

BRENEZ Nicole \& Jonathan LARCHER, 2015, « Romani Cinema. Diffamations figuratives, rectifications documentées », Débordements, 24 Juin 2015, Url : <http://www.debordements.fr/RomaniCinema-1-512>[consulté le 8 août 2016].

CROWE David M., 1994, «Czechoslovakia », A History of the Gypsies of Eastern Europe and Russia, New York, St. Martin's Griffin, pp.31-67.

FOUCAUlt Michel, 2001 [1977], « La vie des hommes infâmes », Dits et écrits 1954-1988, vol. II, édition établie sous la direction de Daniel Defert et François Ewald avec la collaboration de Jacques Lagrange, Paris, Gallimard, p. 237-253.

HAMEs Peter, 2005 [1985], The Czechoslovak New Wave. Second Edition, London/New York, Wallflower Press.

HAMES Peter, 2009, «Slovak Directions », Czech and Slovak Cinema. Theme and Tradition, Edinburgh, Edinburgh University Press, p. 206-228.

HOMER Sean, 2006, «"The Roma Do Not Exist" : The Roma as an Object of Cinematic Representation and the Question of Authenticity ", Gramma: Journal of Theory and Criticis, ${ }^{\circ} 14$, « Objects: Material, Psychic, Aesthetic », p. 183-197.

IORDANOVA Dina, 2006, « Welcome Pictures, Unwanted Bodies. "Gypsy” Representations in New Europe's Cinema », Valentina GLAJAR et Domnica RADULESCU (dir.), “Gypsies” in European Litterature and Culture, New York/Basingstoke, Pilgrave Macmillan, p. 235-240.

LARCHER Jonathan, 2014, « Bohémiens et cinéma fantastique : un imaginaire européen ou des représentations nationales? ", Otrante, n³6, numéro thématique « Le Fantastique de l'Est : Dictatures imaginaires et politiques », p. 91-104.

LARCHER Jonathan, 2017, « ROM. Entretien avec Menelaos Karamaghiolis », Débordements, Url : <http://www.debordements.fr/ROM-vostf> [consulté le 29 mars 2017].

LOSHITZKY Yosefa, 2003, «Quintessential Strangers: The Representation of Romanies and Jews in Some Holocaust Films ", Framework: The Journal of Cinema and Media, vol. 44, nº 2, p. 57-71.

MARCHIORI Dario, 2010, « Trop près, trop loin. Werner Schroeter, une politique de l'exotisme ", Vertigo, $n^{\circ} 38$, p. 99-106. 
PIRSCHTAT Jutta (Hrsg.), 1991, Zeit für Mitteilungen: Peter Nestler, Dokumentarist, Essen, Ed.

Filmwerkstatt.

PócsiK Andrea, 2017, « Screened Otherness: A Media Archaeology of the Romani's

Criminalization », GRZINIĆ Marina, STOJNIĆ Aneta \& Miško sUVAKOVIC (dir.), Regimes of Invisibility in

Contemporary Art, Theory and Culture, New York, Palgrave Macmillan, p. 141-157.

STEWART Michael, 2011 [2010], « Une catastrophe invisible. La Shoah des Tsiganes », STEWART

Michael \& Patrick wiLliams (dir.), Des Tsiganes en Europe, Paris, éditions de la Maison des sciences

de l'homme, p. 249-280.

STEWART Michael, 2014, « Fossoyeurs du sang nordique. Tobias Portschy et l'élaboration d'un racisme anti-tsiganes systématique dans le Burgenland autrichien », CoQUio Catherine \& Jean-Luc POUEYTo (dir.), Roms, Tsiganes, Nomades. Un malentendu européen, Paris, Karthala, p. 99-123.

TEBBUTT Susan, 2003, «Between Distance and Proximity: Film Images and After-images of the Genocide of the Romanies », Framework: The Journal of Cinema and Media, vol. 44, nº 2, p. 72-80. TOUSSAINT Evelyne, 2014, «Le merveilleux est-il l'opium de la pensée critique ? Les images des Tsiganes, Roms et Gitans dans l'imaginaire occidental contemporain », coQUio Catherine \& JeanLuc PoUeYto (dir.), Roms, Tsiganes, Nomades. Un malentendu européen, Paris, Karthala, p. 459-471. WAGENAAR Aad, 2005 [1995], Settela. The Girl Who Got Her Name Back, traduit du néerlandais par Janna Eliot, Nottingham, Five Leaves.

\section{NOTES}

1. Ce mouvement est notamment perceptible dans l'évolution des images photographiques et de la presse illustrée française entre la fin du XIX ${ }^{\mathrm{e}}$ siècle et le début $\mathrm{du} \mathrm{xx}^{\mathrm{e}}$ siècle, voir: Ilsen About, "Une fabrique visuelle de l'exclusion. Photographies des Tsiganes et figures du paria entre 1880 et 1914 ", Catherine Coquio et Jean Luc Poueyto (dir.), Roms, Tsiganes, Nomades. Un malentendu européen, Paris, Karthala, 2014, p. 431-446.

2. Sur l'organisation généalogique, locale, bureaucratique de la "politique tsigane nazie » voir : Henriette Asséo, «Une historiographie sous influence », Catherine Coquio et Jean Luc Poueyto (dir.), op. cit., p. 63-82 ; Michael Stewart, « Une catastrophe invisible. La Shoah des Tsiganes », Michael Stewart \& Patrick Williams (dir.), Des Tsiganes en Europe, Paris, éditions de la Maison des sciences de l'homme, 2011 [2010], p. 249-280.

3. Ce travail s'inscrit dans la continuité de la recherche curatoriale engagée avec Nicole Brenez, qui a abouti à la programmation « Romani cinema », présentée à la Cinémathèque Française en juin et juillet 2016. Mes plus vifs remerciements vont à Maria Komninos, Phaedra Papadopoulou et Ricardo Matos Cabo pour m'avoir partagé ou fait connaitre les films. Toute ma reconnaissance et ma gratitude vont à Nicole Brenez.

4. Dina Iordanova, "Welcome Pictures, Unwanted Bodies. "Gypsy" Representations in New Europe's Cinema », Valentina Glajar, Domnica Radulescu (eds.), "Gypsies" in European Litterature and Culture, New York/Basingstoke, Pilgrave Macmillan, 2006, p. 236.

5. Michel Foucault, "La vie des hommes infâmes ", Dits et écrits 1954-1988. Vol. 2. Édition établie sous la direction de Daniel Defert et François Ewald avec la collaboration de Jacques Lagrange. Paris, Gallimard, 2001 [1977], p. 239-240.

6. Idem, p. 238.

7. Idem, p. 240. 
8. Idem, p. 240.

9. Cette défiance à l'égard d'une « histoire téléologique du film » et le choix d'une « approche non linéaire et centrée sur le contenu propre à l'archéologie des media » caractérisent également les travaux d'Andrea Pócsik qui sont une référence incontournable pour une étude des formes et des fonctionnalités des images filmiques dans l'histoire des Roms et Tsiganes. Voir sur ce point: "Screened Otherness: A Media Archaeology of the Romani's Criminalization », Marina Grzinić, Aneta Stojnić \& Miško Suvaković (eds.), Regimes of Invisibility in Contemporary Art, Theory and Culture, New York, Palgrave Macmillan, 2017, p. 142.

10. Deux caractéristiques partagées par la majorité des films réalisés par des cinéastes slovaques de cette période. Peter Hames, Czech and Slovak Cinema: Theme and Tradition, Edinburgh, Edinburgh University Press, Series Traditions in World Cinema, 2010, p. 206.

11. Sur ce point voir: Jonathan Larcher, «Bohémiens et cinéma fantastique : un imaginaire européen ou des représentations nationales?», Otrante, $n^{\circ} 36$, «Le Fantastique de l'Est: Dictatures imaginaires et politiques », Paris, Éditions Kimé, 2014, p. 91-104.

12. Dans la mesure où il est le seul segment du film qui accorde une place centrale à un personnage tsigane, je ne décrirai que celui-ci.

13. Même si la fidélité aux évènements historiques n'est pas un aspect fondamental du film, la participation des soldats tsiganes dans les armées autrichiennes et hongroises, comme le nombre élevé de déserteurs tchèques et slovaques, sont tous deux attestés par les recherche historiographiques. Voir sur ce point : David M. Crowe, "Czechoslovakia », A History of the Gypsies of Eastern Europe and Russia, New York, St. Martin's Griffin, 1994, p. 42-43.

14. Michael Stewart, op. cit., 2011 [2010], p. 254.

15. Aad Wagenaar, Settela. The Girl Who Got Her Name Back. traduit du néerlandais par Janna Eliot. Nottingham, Five Leaves, 2005 [1995].

16. Dario Marchieri, «Trop près, trop loin. Werner Schroeter, une politique de l'exotisme ", Vertigo, $\mathrm{n}^{\circ} 38,2001$, p. 99.

17. Michael Stewart, op. cit., 2011 [2010], p. 272.

18. Michael Stewart, op. cit., 2011 [2010], p. 276.

19. Preuve s'il en est de l'importance de la parole et de la voix dans la manière dont Peter et Zsóka Nestler ont composé leur film, la monographie allemande qui leur est consacrée, reproduit l'intégralité de la continuité dialoguée, avec dialogues et témoignages. Voir : Jutta Pirschtat (éd.), Zeit für Mitteilungen: Peter Nestler, Dokumentarist, Essen, Ed. Filmwerkstatt, 1991, p. 73-84.

20. Menelaos Karamaghiolis dans: Jonathan Larcher, «ROM. Entretien avec Menelaos Karamaghiolis ", Débordements, 28 mars 2017, <http://www.debordements.fr/ROM-vostf> [consulté le 29 mars 2017].

21. Susan Aasman, «Le film de famille comme document historique », Roger Odin (dir.), Le Film de famille : usage privé, usage public, Paris, Méridiens Klincksieck, 1995, p. 105.

22. Entretien réalisé par l'auteur avec Menelaos Karamaghiolis le 29 septembre 2016.

23. Evelyne Toussaint, «Le merveilleux est-il l'opium de la pensée critique ? Les images des Tsiganes, Roms et Gitans dans l'imaginaire occidental contemporain », Catherine Coquio et JeanLuc Poueyto (dir.), op. cit., 2014, p. 459.

24. Plusieurs séquences des Archives Autrichiennes du Film (Filmarchiv Austria) ont été transférées ou numérisées puis distribuées en VHS ou DVD par les éditions Lex Liszt 12, <http:// www.lexliszt12.at/index.php/video/video-historisches-burgenland-2001-detail> [consulté le 15 mars 2017].

25. Entretien de l'auteur avec David Kleinl, le 31 octobre 2015.

26. Michael Stewart, "Fossoyeurs du sang nordique. Tobias Portschy et l'élaboration d'un racisme anti-tsiganes systématique dans le Burgenland autrichien ", Catherine Coquio et JeanLuc Poueyto (dir.), op. cit., 2014, p. 107.

27. Michael Stewart, op. cit., 2014, p. 109. 
28. Michael Stewart, op. cit., 2014, p. 101.

29. Michel Foucault disait « et le discours qui en naît », op. cit., 2001 [1977], p. 239 et 253.

\section{RÉSUMÉS}

À partir de l'étude de quatre films, collectés dans les franges des arts filmiques et les marges des cinématographies de l'Europe centrale et des Balkans, cet article propose un ensemble de jalons pour une histoire politique et visuelle du "Romani cinema", conçu à l'intersection entre les cinématographies d'avant-garde et les familles romanis (Roms, Tsiganes, Sinti). Les répertoires de gestes mobilisés par ces quatre cinéastes forment à la fois des critiques implacables des conventions figuratives et narratives les plus couramment utilisés par les « gypsy films » et des repères essentiels pour une archéologie des archives filmiques du génocide des Tsiganes.

From the study of four films collected in the fringes of the filmic arts and the margins of cinematography in Central Europe and the Balkans, this article proposes a set of milestones for a political and visual history of "Romani cinema", conceived at the intersection between avantgarde cinematographies and Romani families (Roma, Gypsies, Sinti). The repertoires of gestures mobilized by these four filmmakers form both implacable critiques of the figurative and narrative conventions most commonly used by "gypsy films" and essential references for an archeology of film archives of the genocide of Roma/Gypsies.

\section{AUTEUR}

\section{JONATHAN LARCHER}

Cinéaste et anthropologue, Jonathan Larcher est docteur de l'EHESS (CRAL) et diplômé en études cinématographiques (Sorbonne Nouvelle Paris 3). À partir d'une enquête et de films documentaires réalisés dans les rues tsiganes d'un village roumain, ses recherches et créations explorent les enjeux épistémologiques, politiques et méthodologiques soulevés par les arts et les archives filmiques en anthropologie. Il travaille également à l'élaboration d'une histoire visuelle et critique des imageries par lesquelles les familles roms/tsiganes ont été appréhendées. 